\title{
El estado de la investigación en los Trabajos Fin de Máster en periodismo deportivo en España
}

\author{
The State of Research in Master's Dissertations on Sports \\ Journalism in Spain
}

\author{
Rojas-Torrijos, J. L.' \\ Recibido: 13-10-2020 - Aceptado: 20-01-2021 \\ https://doi.org/10.26441/RC20.1-2021-A14
}

\begin{abstract}
RESUMEN: Este artículo, de carácter exploratorio y descriptivo, realiza un diagnóstico general sobre el estado de la docencia y la investigación en periodismo deportivo dentro de los másteres de la especialidad que ofertan las universidades españolas. Tomando esta radiografía como punto de partida, evalúa en qué medida la producción de los Trabajos Fin de Máster responde a objetivos de investigación o a fines profesionales en función del plan de estudios y perfil de profesorado de esas titulaciones. Para ello se ha procedido a una exhaustiva recogida de datos de los ocho centros universitarios que han impartido másteres de la especialidad en los dos últimos cursos académicos, entre 2017 y 2019. Así se han analizado el volumen, las temáticas y las tipologías de TFM desarrollados. La recogida de datos se ha complementado con cuestionarios y consultas a directores y coordinadores de los másteres objeto de estudio. Los resultados confirman que los TFM en los másteres de periodismo deportivo, al ser todos excepto uno títulos propios y al contar en un alto porcentaje con profesores procedentes del ámbito profesional con menor formación investigadora, apenas inciden en teorías y metodologías ni en temáticas que abran nuevas vías hacia la producción científica en este campo de especialización.
\end{abstract}

Palabras clave: periodismo deportivo; universidad; investigación; máster; TFM; especialización periodística

\begin{abstract}
This exploratory and descriptive article diagnoses the general state of teaching and research in university master programmes in Spain. Taking this approach as starting point, this study analyzes to what extent the dissertations developed in these master degrees are more research-oriented or just produced for professional purposes according to their syllabus and the profile of their teaching staff. To this end, a comprehensive collection of data from the eight universities that offered master programmes in sports journalism between 2017 and 2019 was required. Thus, this study examines the volume, the topics and typologies of dissertations that were defended within those last two academic years. The data collection has been accompanied by questionnaires and inquiries sent to the head professors and supervisors of these degrees. Given that all master programmes on sports journalism except for one are non-official and that a high percentage of their teaching staff work in media outlets, the findings confirm that dissertations are scarcely focused on theories nor research methods, and the addressed issues can hardly open new ways to increase the scientific production in this specific field.
\end{abstract}

Keywords: sports journalism; university; research; master degree; master's dissertation; specialist journalism

\footnotetext{
1 José Luis Rojas Torrijos es periodista con amplia experiencia en gabinetes de comunicación y medios radiofónicos, impresos y digitales. Es Doctor en Periodismo por la Universidad de Sevilla y Profesor Contratado Doctor acreditado a Profesor Titular del Departamento de Periodismo II de la misma universidad. Líneas de investigación: periodismo deportivo, periodismo especializado, innovación en periodismo, ética periodística, uso del lenguaje y libros de estilo. jlrojas@us.es, http://orcid.org/0000-0002-7390-9843
} 


\section{Introducción}

El deporte es un área informativa de gran repercusión social que se ha consolidado en España como una de las tipologías periodísticas más demandadas y que mayores cotas de audiencia alcanza en cada uno de los diferentes soportes. Así lo señala, por ejemplo, el Estudio General de Medios (Año Móvil 2019) de la Asociación para la Investigación de Medios de Comunicación (AIMC), que sitúa a Marca como el diario de mayor difusión con casi 1,7 millones de lectores diarios y a otros tres deportivos (AS, Mundo Deportivo y Sport) entre los nueve primeros. Algo similar acontece en internet, donde, según este mismo informe, Marca es junto con El País la web periodística más visitada y, entre las once con más tráfico, se encuentran también los tres rotativos deportivos antes mencionados.

En el ámbito radiofónico, los programas deportivos copan las principales cifras de audiencia tanto en la franja nocturna intersemanal como en fines de semana, mientras que en televisión las emisiones más vistas de cada año siguen siendo las de temática deportiva. Así, el informe Análisis Televisivo (Barlovento Comunicación, 2019) pone de relieve que 6 de los 10 espacios más vistos del año fueron sobre partidos de fútbol. Según este mismo estudio, además, crece el consumo de contenidos deportivos audiovisuales online y de plataformas deportivas de pago como Movistar LaLiga (la que tiene una mayor cuota de mercado de todos los canales temáticos), Bein Liga o Movistar La Liga de Campeones (Barlovento Comunicación, 2019).

Toda esta pujanza del deporte como fenómeno periodístico, que ha propiciado desde 2008 el nacimiento de numerosos nuevos medios dedicados a esta temática (Manfredi, Rojas-Torrijos y Herranz, 2015; Cabrera, Codina y Salaverria, 2019; Toural y López, 2019), y la consecución de numerosos éxitos logrados en los últimos años por atletas y equipos nacionales, han despertado un significativo interés por este campo por parte en las universidades españolas, cuya oferta educativa sobre asuntos deportivos ha aumentado de manera considerable.

Sin embargo, a pesar de la atracción que genera el deporte, aún no se ha producido la consolidación de los estudios universitarios en periodismo deportivo ni, menos aún, del desarrollo de grupos de investigación que promuevan trabajos académicos sobre esta especialización informativa. De esta forma, esta situación no favorece la consolidación de un corpus de investigación más sólido en la materia en España (Paniagua y Rojas-Torrijos, 2015).

Así, las investigaciones acometidas sobre asuntos deportivos se centran sobre todo en otras áreas como la gestión, el marketing, la fisiología, la historia o la economía del deporte, como consecuencia en gran medida de la creación de centros como el de Estudios Olímpicos de la Universidad Autónoma de Barcelona (UAB), el de Estudios Olímpicos de la Universidad Católica San Antonio de Murcia (UCAM), la Cátedra Real Madrid en la Universidad Europea de Madrid (UEM) o el Sports-Lab en la Universitat Pompeu Fabra en Barcelona, creado en 2015. Hasta ahora ninguno de ellos cuenta con una trayectoria investigadora extensa en temas periodísticos.

Cabe, por tanto, preguntarse por qué en España a día de hoy la consideración académica de los estudios sobre periodismo deportivo y la producción científica en este campo no se corresponden con la relevancia social y cultural de este tipo de contenidos informativos ni de la temática sobre la que versa, el deporte y sus competiciones. Asimismo, cabe plantear si esta situación tiene que ver no solo con el grado de implantación de esta área de conocimiento en los planes docentes de Grado, sino también con la estructura, contenidos y profesorado de los másteres existentes, que es lo que puede determinar finalmente qué tipo de trabajos de investigación puedan desarrollarse en el marco de esos títulos de posgrado. 


\section{Metodología}

Con el fin de tratar de confirmar nuestra hipótesis de punto de partida y comprobar los planteamientos arriba formulados, nos proponemos los siguientes cinco objetivos de investigación:

(1) Hacer un diagnóstico general sobre la implantación de la asignatura Periodismo Deportivo dentro de los planes de estudios de los Grados de Periodismo de las universidades españolas y la producción resultante de Trabajos Fin de Grado (TFG) sobre esta materia.

(2) Conocer los planes docentes en los másteres de periodismo deportivo de universidades españolas, así como los perfiles de su profesorado.

(3) Analizar la relación entre esos planes y perfiles docentes con los Trabajos Fin de Máster (TFM) defendidos en los últimos años en universidades españolas que ofrecen alguna de estas titulaciones.

(4) Recopilar y clasificar los TFM defendidos por categorías temáticas y formales (trabajo de investigación o proyecto emprendedor), así como identificar principales líneas de investigación en este campo.

(5) Evaluar en qué medida la producción de TFM en estos másteres responde a objetivos de investigación y si desde los posgrados se puede contribuir a impulsar la producción científica sobre periodismo deportivo en España.

Para dar cumplida respuesta a los objetivos de investigación, la realización de este estudio consta de dos fases bien diferenciadas. Para dibujar el contexto en el que ha de enmarcarse este estudio, en la primera se ha procedido a una exhaustiva recogida de datos de los centros universitarios españoles que imparten la titulación de Grado de Periodismo y, dentro de esos planes de estudio, de las universidades que ofertan la asignatura de Periodismo Deportivo. Además, una segunda fase se ha fundamentado en hacer una radiografía del estado de la investigación dentro de los másteres universitarios de la especialidad que se imparten en España.

En lo que concierne a la primera fase de análisis, ha sido preciso realizar una radiografía para conocer la situación actual de la asignatura dentro de los planes de estudio de Grado en Periodismo y Comunicación en las universidades españolas. Para ello se ha acudido a dos fuentes: por un lado, la Asociación Española de Universidades con Titulaciones de Información y Comunicación (ATIC) y, por otro, el Registro de Universidades, Centros y Títulos (RUCT) del Ministerio de Ciencia, Innovación y Universidades. Posteriormente, se han consultado pormenorizadamente todos los planes de estudio existentes en esta área de conocimiento a través las páginas web de cada una de las universidades.

Una vez delimitadas las titulaciones de Grado que incluyen la asignatura de Periodismo Deportivo, se ha recabado la producción resultante de Trabajos Fin de Grado (TFG) sobre esta materia defendidos en universidades españolas desde la implantación del Espacio Europeo de Educación Superior (Plan Bolonia) en el curso académico 2010-2011 hasta finales de 2019. De esta forma, se ha evaluado el interés que suscita esta temática en unos trabajos que pueden llegar a constituir en muchos casos las primeras aproximaciones a la investigación en este campo desde las facultades. Con este propósito, se han monitorizado las referencias en Google Scholar y se han identificado todos los TFG sobre periodismo deportivo que se encuentran accesibles y se pueden consultar íntegramente en línea en los repositorios digitales de las diferentes universidades. Como consecuencia de este trabajo, se ha actualizado la base de datos de Trabajos Fin de Grado construida en el blog académico Periodismo Deportivo de Calidad (Rojas-Torrijos, 2019). 
Hay que aclarar que, si bien esta relación de TFG no constituye una totalidad de la producción de este tipo de trabajos en el conjunto de titulaciones de Periodismo a nivel nacional al no estar toda ella disponible en línea, sí puede considerarse una muestra representativa. Por un lado, abarca la producción registrada digitalmente de 18 instituciones académicas, prácticamente la mitad del total de las que ofertan el Grado de Periodismo en España. Por otra parte, recoge un número de trabajos amplio y diverso que permite extraer una visión panorámica sobre cuáles son las temáticas de mayor interés en los TFG, que pueden considerarse como las primeras aproximaciones a la investigación sobre esta materia desde las facultades.

Después de fijar las bases de los estudios de Periodismo Deportivo y de los primeros intentos investigadores sobre la materia que se han hecho a la conclusión del Grado de Periodismo, se ha diseñado una segunda fase de investigación centrada en el análisis de los másteres que existen sobre esta especialización en las universidades españolas y se han celebrado en los dos últimos cursos académicos completados previos al desarrollo de esta investigación (2017-2018 y 2018-2019). De esta forma, se pretende conocer cuál es el estado de la investigación en periodismo deportivo dentro de estos posgrados, concretamente el volumen, las temáticas y las tipologías de Trabajos Fin de Máster (TFM) desarrollados.

Para ello se ha utilizado también el RUCT, así como el censo de másteres sobre esta especialidad que recoge el blog Periodismo Deportivo de Calidad (Rojas-Torrijos, 2017), elaborado a partir del análisis de contenido de las webs oficiales de cada uno de los programas. De esta forma, se ha podido conocer no solo el número de másteres existentes, los centros donde se imparten y su localización geográfica, sino también las modalidades de posgrado, los planes de estudio (módulos y principales materias que se imparten) e incluso los perfiles docentes de los claustros. Asimismo, se ha analizado la relación entre esos planes y perfiles docentes con las tipologías y temáticas de los Trabajos Fin de Máster defendidos. La recogida de datos se ha complementado con cuestionarios enviados por correo electrónico a profesores responsables de los másteres objeto de análisis con el propósito de validar los datos obtenidos y ampliarlos desde un punto de vista cualitativo.

Dentro de la oferta de másteres existente, ha sido preciso también averiguar cuáles de esos másteres son oficiales, de forma que podamos seleccionar aquellos que, además de trazar una línea de empleabilidad enfocada a una formación puramente profesional, ofrecen la posibilidad de continuar en la Universidad profundizando en nuevas líneas de investigación sobre la materia. A partir de este diagnóstico de los estudios de Grado y Posgrado sobre periodismo deportivo en España, analizamos en qué medida la producción de TFM en estos másteres responde a objetivos de investigación o de empleabilidad y si desde los posgrados se puede contribuir realmente a impulsar la producción científica y la transferencia de conocimiento y especialización en este campo informativo.

Para validar y ampliar los datos recabados desde un punto de vista cualitativo, a lo largo de la investigación se han enviado cuestionarios por correo electrónico y se han realizado varias consultas por vía telefónica a las personas responsables de la dirección y coordinación de cada una de estas titulaciones. Se obtuvieron respuesta en 7 de los 8 másteres estudiados. Las personas que atendieron las peticiones de información para este estudio fueron: Javier Vidaurreta (UEM), Sergi Cortiñas y Pablo Rausell (Universidad Pompeu Fabra), Arantxa Román (Universidad de Sevilla), Jordi Colomé (Universidad Ramón Llull), Pedro Luis Pérez (UCAM), Antonio Vaquerizo (Universidad Nebrija) y Juan Carlos Nieto y Juan Castro (CEU San Pablo-Marca).

\section{Resultados}

Tal como han señalado diversos estudios, en los últimos años se ha experimentado un interés creciente en España por los estudios de Comunicación y Periodismo, que ya se ofrecen en más de cuarenta uni- 
versidades (Pestano, Rodríguez y Del Ponti, 2011). No obstante, es preciso delimitar entre estas dos áreas de conocimiento antes de analizar cuáles son el grado de implantación y la relevancia adquirida por el Periodismo Deportivo como materia en los estudios de Grado universitario en este país.

\subsection{Situación de la asignatura de Periodismo Deportivo en las titulaciones de Grado}

La Asociación Española de Universidades con Titulaciones de Información y Comunicación (ATIC) está integrada en la actualidad por 36 universidades (38 facultades). A esas 36 instituciones académicas resultantes hay que sumar otras 13 universidades que no aparecen en la referida asociación pero que sí ofertan de manera oficial estas titulaciones, tal como consta en el RUCT, con datos de febrero de 2020. Se trata de 8 universidades privadas (UCAM, Europea de Madrid, Loyola Andalucía, Camilo José Cela, Francisco de Vitoria, Fernando Pessoa Canarias, Abat Oliba CEU (Barcelona) y Europea del Atlántico (Santander) y 5 universidades públicas (Miguel Hernández, La Laguna, Cádiz, Politécnica de Valencia y Extremadura, que lanzó el Grado de Periodismo en 2018).

En esta lista no se han incluido los centros adscritos a universidades públicas (Centro Universitario EUSA, de la Universidad de Sevilla; Centro Universitario Villanueva, de la Universidad Complutense; o ESIC, de la Universidad Rey Juan Carlos), ya que constituyen una duplicación de los planes de estudios existentes respecto a las universidades principales de las que dependen académicamente.

Del total de 49 universidades españolas que encontramos con titulaciones de Información y Comunicación, no hay Grado de Periodismo en 12 (Tabla 1): Alicante (grado de Publicidad y Relaciones Públicas), Vigo (grados de Publicidad y Relaciones Públicas y de Comunicación Audiovisual), Granada (grados de Comunicación Audiovisual y de Información y Documentación), Coruña (Comunicación Audiovisual), Lleida (Comunicación y Periodismo Audiovisual), Universidad de Barcelona (Comunicación e Industrias Culturales o en otra Facultad, Comunicación Audiovisual y/o Gestión de la Información y la Documentación Digital son los grados), Salamanca (USAL) (Grados de Comunicación Audiovisual y de Comunicación Creación Audiovisual), Universitat Oberta de Catalunya-UOC (Grado de Comunicación), Politécnica de Valencia (Comunicación Audiovisual en campus de Gandía), Cádiz (Publicidad y RR.PP. en Jerez de la Frontera), Loyola Andalucía (Grado de Comunicación) y Camilo José Cela (Grado de Comunicación).

Por tanto, en la actualidad se puede cursar el Grado de Periodismo en 37 universidades españolas. Esto representa un ligero incremento respecto a los datos recogidos en estudios anteriores, como el de Sánchez-García (2016), que hablaba de 35 centros con oferta reglada de estudios de Periodismo, y una idéntica cifra a la registrada por Torregrosa (2015). Esta progresión también se observa en el cómputo total de titulaciones al sumarse los Grados de Comunicación, Comunicación Audiovisual y Publicidad y Relaciones Públicas, que han pasado de 42 (Cabezuelo y Sotelo, 2014) a los 49 indicados en la presente investigación.

Tabla 1. Titulaciones universitarias en Periodismo en España

\begin{tabular}{|l|c|c|c|}
\hline \multicolumn{1}{|c|}{ Estudios } & \multicolumn{1}{c|}{ Nentros } & 49 & $100 \%$ \\
\hline Titulaciones de Comunicación e Información & 49 & \\
\hline & Universidades públicas & 30 & Porcentaje \\
\hline & Universidades privadas & 19 & \\
\hline Grados de Periodismo & 37 & $75,5 \%$ \\
\hline & Universidades públicas & 21 & \\
\hline \multicolumn{3}{|c|}{ Fuente: elaboración propia. } \\
\hline
\end{tabular}


Tras analizar el contenido de los planes de estudio de las 37 universidades con titulación de Periodismo, se observa que la asignatura de Periodismo Deportivo no está presente en 17 de esas carreras, es decir, en casi un $46 \%$ de los casos (Tabla 2). Así no consta en la programación de las universidades de: Santiago de Compostela, Málaga, País Vasco, Murcia, Rovira i Virgili, San Jorge, Rey Juan Carlos, Nebrija, Ramón Llull, La Laguna, Europea del Atlántico y Extremadura. En estos casos ni siquiera aparece mencionada como opción y casi siempre aparece integrada, muy tangencialmente, dentro de la asignatura obligatoria de Periodismo Especializado, presente esta última en la mayoría de los planes de estudios de la titulación.

Tabla 2. La asignatura de Periodismo Deportivo

\begin{tabular}{|l|c|c|}
\hline Estudios & Número & Porcentaje \\
\hline Grados de Periodismo & 37 & $100 \%$ \\
\hline Grados sin asignatura de Periodismo Dvo. & 17 & $45,9 \%$ \\
\hline Grados con asignatura de Periodismo Dvo. & 20 & $54,1 \%$ \\
\hline Universidades públicas & 12 & \\
\hline Universidades privadas & 8 & \\
\hline
\end{tabular}

Fuente: elaboración propia.

En otros planes de estudios sí hay menciones de la materia, aunque de forma adyacente a la titulación: como asignatura de la Mención en Periodismo Especializado en Udima (Universidad a Distancia de Madrid); como parte del nombre de la asignatura en la que se engloba, Periodismo Especializado I: Científico, Económico y Deportivo, en la UCAM o Periodismo Especializado I (Introducción al Periodismo Especializado, Periodismo Deportivo y Periodismo Tecnológico) en Abat Oliba CEU; como doble mención de Título de Experto en Periodismo Deportivo, desplegada en dos optativas en los dos últimos cursos del Grado (El Deporte sobre el Papel, El Diseño de la Comunicación Deportiva, Comunicación de Eventos Deportivos; e Infografía Deportiva, El Deporte sube a la Red) en la Cardenal Herrera CEU; y como Título de Experto en Periodismo Deportivo de forma simultánea al Grado, en la San Francisco de Vitoria.

Por tanto, Periodismo Deportivo expresamente solo aparece como asignatura de especialización en los planes de estudios de Grado de 20 universidades (54,1\% del total), seis más que anteriores estudios (Elías y Luengo, 2014), lo que la consolida como la tercera tipología informativa más frecuente en los currículos docentes tras Periodismo Político y Económico. Las universidades que ofertan la asignatura son: Valencia, Valladolid, Zaragoza, Sevilla, CEU San Pablo, Miguel Hernández (UMH), Pontificia de Salamanca (UPSA), Pompeu Fabra, Navarra, Jaume I, Complutense (aquí se llama "Periodismo Especializado en Educación y Deporte"), Europea Miguel de Cervantes (UEMC), Castilla-La Mancha (UCLM), Carlos III, Autónoma de Barcelona (UAB), Europea de Madrid (UEM), CESAG-Comillas, Vic, Internacional de Catalunya (UIC) y Fernando Pessoa Canarias.

En la mayoría de los casos (ver Tabla 3) se trata de una materia optativa de tercer o cuarto curso, con raras excepciones como la Universidad Europea de Madrid, donde es obligatoria de tres créditos en Tercero y se llama "Deporte y Comunicación" o en CESAG, donde es optativa de tres créditos y lleva el nombre de "Deportes y medios de comunicación". 
Tabla 3. La asignatura Periodismo Deportivo en los planes de estudio

\begin{tabular}{|l|c|l|c|}
\hline Universidad & Tipo de asignatura & Curso & Créditos ECTS \\
\hline Valencia & Optativa & $4^{\circ}$ & 4,5 \\
\hline Valladolid & Optativa & $4^{\circ}$ & 6 \\
\hline Zaragoza & Optativa & $3^{\circ}-4^{\circ}$ & 6 \\
\hline Sevilla & Optativa & $3^{\circ}$ & 6 \\
\hline CEU San Pablo & Optativa & $3^{\circ}-4^{\circ}$ & 6 \\
\hline UMH & Optativa & $4^{\circ}$ & 6 \\
\hline UPSA & Optativa & $4^{\circ}$ & 3 \\
\hline Pompeu Fabra & Optativa & $3^{\circ}-4^{\circ}$ & 4 \\
\hline Navarra & Optativa & $3^{\circ}-4^{\circ}$ & 3 \\
\hline Jaume I & Optativa & $4^{\circ}$ & 6 \\
\hline Complutense & Optativa & $4^{\circ}$ & 6 \\
\hline UEMC & Optativa & $4^{\circ}$ & 6 \\
\hline UCLM & Optativa & $4^{\circ}$ & 6 \\
\hline Carlos III & Optativa & $3^{\circ}-4^{\circ}$ & 6 \\
\hline UAB & Optativa & $4^{\circ}$ & 6 \\
\hline UEM & Obligatoria & $3^{\circ}$ & 3 \\
\hline CESAG & Optativa & $3^{\circ}$ & 3 \\
\hline Vic & Optativa & $4^{\circ}$ & 6 \\
\hline UIC & Optativa & $3^{\circ}-4^{\circ}$ & 6 \\
\hline F. Pessoa & Optativa & $3^{\circ}-4^{\circ}$ & 6 \\
\hline
\end{tabular}

Fuente: elaboración propia.

\subsubsection{Caracterización de los Trabajos Fin de Grado sobre periodismo deportivo}

Una vez radiografiada la realidad del Periodismo Deportivo dentro de los planes de estudios universitarios vigentes, se ha recabado la producción resultante de Trabajos Fin de Grado (TFG) sobre esta materia defendidos en universidades españolas desde la implantación del Espacio Europeo de Educación Superior (Plan Bolonia) en el curso académico 2010-2011 hasta finales de 2019.

El propósito de esta compilación es evaluar el interés que despierta esta área especializada entre los estudiantes al tratarse de unos trabajos que en muchos casos y según la tipología elegida, pueden considerarse como las primeras aproximaciones a la investigación sobre esta materia desde las facultades. En este sentido los TFG pueden tener rasgos comunes con otros trabajos de titulaciones superiores como los de fin de máster o las tesis doctorales (Díaz-Vázquez, García-Díaz, Maside y Vázquez-Rozas, 2018: 161).

Como se indica en el Real Decreto 1993/2007, por el que se establece la ordenación de las enseñanzas universitarias oficiales, el TFG es una asignatura que debe estar orientada a la evaluación de competencias asociadas al título. A partir de esta norma, cada universidad cuenta con su propia regulación de los Trabajos Fin de Grado.

Los estudiantes de Grado de Periodismo suelen optar así entre dos grandes modalidades a la hora de hacer su trabajo o proyecto fin de carrera: o bien se trata de un proyecto de investigación que aborde una temática original y relevante, y se desarrolle de acuerdo a la metodología científica adecuada; o bien se ofrece la posibilidad de hacer un trabajo de carácter experimental o profesional (reportaje, reportaje audiovisual o diseño de un proyecto periodístico suelen ser los más 
habituales) que deberá acompañarse de una memoria que recoja el proceso de elaboración y planificación del trabajo.

Según los TFG de Periodismo defendidos entre 2012 y 2019 y disponibles en acceso abierto en los repositorios digitales de las universidades españolas, se han censado 288 trabajos provenientes de 18 instituciones académicas, esto es, el 48,6\% del total de los centros que ofertan el Grado de Periodismo. De esos 288 trabajos, apenas $32(11 \%)$ son de índole práctica, una tipología cada vez más frecuente en los últimos cursos académicos. Como dato curioso, llama la atención que haya TFG sobre periodismo deportivo en algunas universidades que no ofertan esa asignatura en sus planes de estudio de Grado, como las de Málaga (3), País Vasco (2), Rey Juan Carlos (2) o, muy especialmente, La Laguna, con 28 trabajos presentados sobre esta temática en los últimos ocho años.

Por tanto, en estos TFG de Periodismo que versan sobre la especialización en materia deportiva prevalece la tipología de trabajo de investigación. Al tratarse de estudios tempranos en este campo, es oportuno analizar también las temáticas abordadas, con vistas a su posible continuación en investigaciones posteriores. De esta manera, predominan sobre todo tres: los estudios del periodismo deportivo desde una perspectiva de género (tratamiento informativo desigual de las mujeres deportistas o aspectos del lenguaje inclusivo en deportes), el infoentretenimiento en los programas deportivos en televisión y la gestión de comunicación deportiva en redes sociales tanto en coberturas informativas como desde los gabinetes de los principales clubes de fútbol en España. Otros aspectos tratados, aunque con menor frecuencia que los anteriores, son: estudios de caso de emprendimientos y nuevos medios periodísticos surgidos en estos últimos años, la cobertura de deportes "minoritarios" o menos mediáticos, el uso de nuevas narrativas y formatos digitales, y cuestiones historiográficas del periodismo deportivo.

\subsection{Caracterización de los Trabajos Fin de Máster sobre periodismo deportivo}

Entretanto, en el ámbito de Posgrado los estudios sobre Periodismo han alcanzado un notable desarrollo en los últimos años en España. Así, durante el curso 2012-2013 se ofrecieron 57 programas de doctorado y 135 títulos de Máster. De estos últimos, el 21\% (57 títulos) corresponden al área de Comunicación, sumando los de Periodismo, Publicidad y Comunicación (Castillo et al., 2013). Según los datos analizados en el RUCT, en la actualidad hay 26 másteres oficiales verificados de Periodismo, los cuales se imparten en centros universitarios de las comunidades autónomas de Madrid (16), Cataluña (4), Comunidad Valenciana (2), y La Rioja, País Vasco, Andalucía y Galicia (1).

Tabla 4. Másteres universitarios de Periodismo Deportivo en España

\begin{tabular}{|l|l|l|l|c|}
\hline Universidad & Ciudad & Modalidad & Tipología & Ediciones \\
\hline UEM & Madrid & Presencial & Máster Oficial & 14 \\
\hline Ramón Llull & Barcelona & Presencial & Título Propio & 9 \\
\hline Pompeu Fabra & Barcelona & Presencial & Título Propio & 8 \\
\hline CEU San Pablo & Madrid & Presencial/online & Título Propio & $8 / 12^{*}$ \\
\hline Sevilla & Sevilla & Presencial & Título Propio & 8 \\
\hline Villanueva & Madrid & Presencial/online & Título Propio & $7 / 3^{\star}$ \\
\hline UCAM & Murcia & Online & Título Propio & 3 \\
\hline Nebrija & Madrid & Presencial & Título Propio & 3 \\
\hline
\end{tabular}

Fuente: elaboración propia. *En primer lugar, aparece el número de edición presencial y luego la online.

En lo que respecta al campo del Periodismo Deportivo, hoy en día son 8 universidades españolas las que ofrecen un título de posgrado (ver Tabla 4). De ellas, 6 son privadas (Nebrija, UEM, 
UCAM, Ramón Llull, CEU San Pablo y Centro Universitario Villanueva, aunque adscrito a la Complutense) y 2 públicas (Sevilla y Pompeu Fabra). Si atendemos a su distribución geográfica, también aquí la mayor parte se concentra en Madrid (4), seguida de Cataluña (2) y Andalucía y Murcia (1). De todas estas titulaciones solo una es oficial: Universidad Europea de Madrid, que ya marcha por su decimocuarta edición e incluso cuenta con una modalidad en lengua inglesa. Con anterioridad adquirieron la condición de oficial solo los másteres de la especialidad ofertados por la Universidad Camilo José Cela (hasta 2018) y la Universidad Pontificia de Salamanca (hasta 2017) $)^{2}$.

Una característica de la mayor parte de estos másteres reside en el hecho de que las universidades se alían con medios de comunicación y clubes para coorganizar o lograr patrocinios que cofinancian su desarrollo. Así, la Universidad de Sevilla lleva a cabo su título propio en colaboración con el Sevilla Fútbol Club, una cooperación que sigue el modelo del máster oficial de la Universidad Europea de Madrid (de la mano del Real Madrid) o el título propio de la Universitat Ramón Llull (con el Fútbol Club Barcelona).

Igualmente, la Universidad CEU San Pablo viene impartiendo su título de especialización en Periodismo Deportivo junto con el diario Marca, tanto en su modalidad presencial como en la online, mientras que la Universidad Nebrija ofrece su Máster en Periodismo y Retransmisiones Deportivas en colaboración con la plataforma televisiva Movistar + .

También aquí cabe establecer posibles paralelismos de la oferta entre facultades que cuentan con la asignatura de Periodismo Deportivo dentro de los estudios de Grado y aquellas que ofertan un máster en esta precisa materia. En este caso, de las 8 universidades que imparten posgrados de la especialidad, hay 3 (Nebrija, Ramón Llull y UCAM) que no ofrecen la asignatura en sus programas de Grado.

Esto supone que las titulaciones de máster no dan continuidad al ciclo de enseñanza superior anterior y que son autónomas respecto a la oferta académica global de la Universidad. Además, y atendiendo a la clasificación de másteres que propone Haug (2015), puede entenderse que la decisión de estas universidades de ofertar un máster de este tipo responde más objetivos a profesionalizantes y prácticos que a los de contribuir a una verdadera superespecialización, que, por el contrario, se lograría profundizando en unos contenidos planteados ya desde los estudios de Grado con el fin de corresponder mejor a tareas y perfiles que se demandan en el mercado laboral. Además, el hecho de que solo haya un máster oficial limita mucho las posibilidades de crear vertientes investigadoras en este campo a nivel nacional.

Atendiendo al análisis de contenido de los programas de los 8 másteres de Periodismo Deportivo, existe una gran diversidad en cuanto a la distribución de módulos y materias. Se observan pocos contenidos relacionados con la cultura deportiva (las que más inciden en ello son las dos universidades públicas, Sevilla y Pompeu Fabra); el número de modalidades abordadas no es muy extenso (sobre todo fútbol y deportes más mediáticos); prima lo audiovisual hasta el punto de llegar a copar el temario (ocurre en los de Nebrija y Villanueva); apenas aparece el inglés para periodistas deportivos (la Pompeu ofrece un taller de locución específico mientras que la UEM imparte una modalidad de su máster en esta lengua); y cobra cada vez más importancia la gestión de comunicación en clubes (UCAM, UEM, Sevilla o CEU San Pablo-Marca).

\footnotetext{
${ }^{2}$ De esta lista se han dejado fuera otros centros de enseñanza privada no universitarios que también ofertan posgrados de especialidad, tales como la Escuela de Negocios de la Cámara de Comercio de Sevilla o CES-Escuela Superior de Imagen y Sonido en Madrid.
} 


\subsubsection{Perfil de profesorado y posibilidades de tutorización de TFM}

Respecto a los perfiles del profesorado de los másteres en Periodismo Deportivo que se imparten en universidades españolas (ver Tabla 5), se observan notables diferencias entre las titulaciones ofertadas. Dichas variaciones están relacionadas con el enfoque más o menos profesionalizante o de investigación que haya adoptado cada máster. Asimismo, la prevalencia de un cierto perfil de profesor en relación a otros puede tener incidencia en la tipología o el modelo de Trabajo Fin de Máster que finalmente se lleva a cabo dentro de cada titulación.

Tabla 5. Perfil de profesorado de Másteres Periodismo Deportivo

\begin{tabular}{|l|c|c|c|}
\hline Universidad & Total profesores & Tutores de TFM & \% Tutores TFM doctores \\
\hline Sevilla & 45 & 21 & $100 \%$ \\
\hline UEM & 53 & 26 & $78 \%$ \\
\hline UCAM & 22 & 12 & $75 \%$ \\
\hline Pompeu Fabra & 19 & 11 & $63 \%$ \\
\hline CEU San Pablo & 27 & 27 & $15 \%$ \\
\hline Nebrija & 17 & 17 & $12 \%$ \\
\hline Ramón Llull & 41 & 41 & $5 \%$ \\
\hline Villanueva & 27 & 27 & $0 \%$ \\
\hline
\end{tabular}

Fuente: elaboración propia.

Si bien el total de profesores participantes oscila entre los 17 del claustro del máster en la Universidad de Nebrija y los 53 en el de la UEM, en esta última se establece una clara diferencia entre speakers, es decir, aquellos profesionales colaboradores que solo dan unas pocas horas y profesorado, interno pero también externo, que tiene un perfil más académico. Esto significa que el número de profesores que pueden dirigir un TFM es muy inferior al del total.

Igualmente acontece, por ejemplo, en el título propio de la Universidad de Sevilla. En este caso, de los 45 profesores solo 21 pueden acometer las tareas de tutorización porque para ello se precisa que sean doctores. En el supuesto de que un estudiante quiera hacer un trabajo que requiera una orientación más práctica o experimental, se recurre a quienes conforman el profesorado profesional, que actúan como asesores sin la posibilidad de cotutorizar. En la Universidad Pompeu Fabra, entretanto, la mayoría de los profesores que pueden dirigir un TFM son doctores, pero también pueden serlo otros perfiles con experiencia o trayectoria dando clases en la institución.

Pese a que, en general, el número de académicos y doctores en el profesorado de estas titulaciones es visiblemente inferior al de los profesionales que proceden de medios de comunicación, la mayor parte de estos másteres conjugan ambos perfiles, también para labores de tutorización. Así, por ejemplo, lo hace la Universidad CEU San Pablo en su máster con Marca, donde cualquier miembro de su profesorado puede ser tutor de TFM, de forma que la asignación a un perfil o a otro siempre responda a la naturaleza del trabajo. El perfil más académico suele ocuparse de trabajos de investigación, mientras que los periodistas serán los que supervisen proyectos más prácticos.

Dada esta configuración tan variada en cuanto a planes de estudios y a perfiles docentes dentro de la oferta de másteres en periodismo deportivo en universidades españolas, a continuación nos detenemos en analizar posibles relaciones entre la naturaleza, la estructura de profesorado y el enfoque temático adoptado por estas titulaciones con los Trabajos Fin de Máster defendidos dentro de estas titulaciones en los dos últimos cursos académicos previos a la realización de este estudio, 20172018 y 2018-2019. 


\subsection{Análisis de los Trabajos Fin de Máster sobre periodismo deportivo (2017-2019)}

A la hora de analizar los TFM presentados entre 2017 y 2019 en los ocho másteres en periodismo deportivo que ocupan esta investigación, en primer lugar, hemos preguntado a los responsables de esas titulaciones por el procedimiento que recoge la normativa de cada universidad para la elaboración, tutorización y defensa de estos trabajos o proyectos.

Tabla 6. Número y tipología de TFM en Másteres Periodismo Deportivo

\begin{tabular}{|l|c|c|c|c|}
\hline Universidad & \multicolumn{1}{|c|}{ Total TFM } & \multicolumn{1}{c|}{ Modalidad } & \multicolumn{1}{l|}{ Investigación } & Práctico \\
\hline CEU San Pablo & 37 & Individual & 15 & 22 \\
\hline Sevilla & 18 & Individual & 13 & 5 \\
\hline UEM & 15 & Colectivo & 6 & 9 \\
\hline UCAM & 13 & Individual & 13 & 0 \\
\hline Total & 83 & $47(56,6 \%)$ & $36(43,4 \%)$ & \\
\hline
\end{tabular}

Fuente: elaboración propia.

De esta forma, los trabajos pueden realizarse, según el caso, a título individual o de manera colectiva y pueden englobarse en dos grandes tipologías de TFM: los llamados de trabajos investigación o académicos, que siguen una metodología y analizan un aspecto concreto o caso/s de la realidad mediática del deporte; y los trabajos prácticos, que suelen ir desde el reportaje de largo aliento (investigación, histórico o en profundidad sobre un tema de calado) o el reportaje audiovisual hasta el proyecto empresarial o la creación de un producto periodístico concreto en materia deportiva. En cinco casos (CEU San Pablo, Sevilla, UEM. Pompeu Fabra y UCAM), la normativa permite al alumnado optar por una modalidad u otra, mientras que Nebrija, Villanueva y Ramón Llull el TFM es de naturaleza puramente práctica.

Así, por ejemplo, en el máster de la Universidad Ramón Llull los estudiantes desarrollan un proyecto periodístico. Entre las iniciativas desarrolladas en los últimos cursos, sobresalen proyectos para la creación de webs y podcasts superespecializados, así como el planeamiento para la puesta en marcha de empresas de comunicación, tales como productoras de contenido deportivo o incluso agencias de representación de deportistas.

Por su parte, en la Universidad Nebrija el objeto del TFM es la realización de un informativo de deportes en televisión de creación propia y consiste en un proyecto colectivo de entre 4 y 5 miembros. Aunque se hace por grupos, la normativa de esta última titulación señala que el trabajo deberá acompañarse de una memoria individual que habrá de reunir los estándares de calidad de estilo, redacción y citación bibliográfica similares a los de un trabajo académico.

En lo que respecta a las tipologías que coexisten dentro de un mismo máster (Tabla 6), el 56,6\% de los TFM presentados en el periodo analizado se corresponde con trabajos de investigación y un $43,4 \%$ de proyectos son de índole más experimental o creativa. Por universidades, UCAM y Sevilla priorizan la investigación frente a UEM y CEU San Pablo, en las que, en consonancia con su naturaleza (se hacen con el Real Madrid y con el diario Marca, respectivamente), son más numerosos los trabajos prácticos.

En el periodo que abarca esta investigación, la Universitat Pompeu Fabra todavía no había cosechado resultados, dado que la implantación del TFM en su normativa para este máster en concreto se haría efectiva en el curso 2019-2020. Según indican desde la dirección de este ciclo formativo, los TFM podrán ser o bien trabajos académicos o bien reportajes que los estudiantes deberán entregar junto a una memoria. Dentro de la modalidad práctica, los reportajes podrán ser de investigación, histórico o un gran reportaje sobre un tema relevante. 
Tabla 7. Temáticas de TFM de investigación

\begin{tabular}{|l|r|r|}
\hline Temática & 12 & $25,5 \%$ \\
\hline Fútbol / Fútbol y TV & 11 & $23,4 \%$ \\
\hline Deporte femenino & 4 & $8,5 \%$ \\
\hline Deporte y política & 4 & $8,5 \%$ \\
\hline Redes sociales & 3 & $6,4 \%$ \\
\hline Nuevas tendencias en Periodismo deportivo & 3 & $6,4 \%$ \\
\hline Dopaje & 2 & $4,3 \%$ \\
\hline Arbitraje & 8 & $17 \%$ \\
\hline Otros & 47 & $100 \%$ \\
\hline Total & & \\
\hline
\end{tabular}

Fuente: elaboración propia.

Además de clasificar los TFM defendidos en estos másteres por categoría formal, se han identificado las temáticas más habituales sobre las que han versado estos trabajos. Tal como se aprecia en la Tabla 7, predominan los que están relacionados con el fútbol (derechos de televisión, competiciones internacionales, marketing) y el deporte femenino (fútbol, pero también otros deportes como gimnasia o rugby, gestión y arbitraje).

Otros trabajos de investigación inciden en aspectos de política e historia del deporte, redes sociales (Youtube, Instagram, Twitter), nuevas tendencias en el periodismo deportivo (periodismo de datos, fotoperiodismo, la evolución de la narración de partidos), casos de dopaje en el deporte (con especial incidencia en el ciclismo), audiencias de televisión, nuevas relaciones del periodista con sus fuentes más habituales, así como estudios de caso sobre el tratamiento informativo que reciben determinados protagonistas (Rafa Nadal, Pep Guardiola), equipos o deportes en los medios.

\section{Conclusiones y discusión}

A tenor de los resultados obtenidos tanto en el proceso de recogida de datos como en el de consultas y cuestionarios realizados, se pone de manifiesto la necesidad de potenciar los estudios sobre Periodismo Deportivo en las universidades españolas y de ampliar la producción científica desde los Trabajos Fin de Máster en las titulaciones de posgrado sobre esta materia que existen en España.

Por un lado, resulta llamativo que, a pesar de que Periodismo Deportivo solo está presente en poco más de la mitad de los planes de estudios de los Grados de Periodismo que ofertan las universidades españolas, el interés por estos estudios se refleja en un número relevante de TFG de investigación sobre la materia que se defienden cada año aún en varios centros académicos donde no se imparte la asignatura. Los TFG constituyen así aproximaciones al estudio científico que permiten cultivar una cantera de investigadores en los departamentos de las diferentes facultades.

Por otra parte, el estudio señala que la producción de TFM en los másteres existentes en España sobre esta especialización no responde a objetivos de investigación precisamente porque todos excepto uno son títulos propios, de naturaleza profesionalizante y con fines de empleabilidad, y apenas incluyen módulos formativos sobre teorías y metodologías investigadoras en sus planes de estudio.

No obstante, esta carencia se suple en muchos casos con una presencia mayoritaria, dentro del profesorado de cada máster, de tutores de trabajos que poseen el grado de doctor. Esta situación favorece que los estudiantes decidan, en más de un 56\% de los casos, por la realización de un TFM en la modalidad de investigación antes que proyectos y trabajos de carácter periodístico o de emprendimiento. 
El hecho de que solo haya un máster oficial (UEM), que sí mantiene una vertiente de formación investigadora, limita que desde estas titulaciones pueda impulsarse la producción científica sobre periodismo deportivo en España. No solo no se abre suficientemente el camino directo a estudios de doctorado que den continuidad a las líneas de investigación trazadas desde los TFM, sino que tampoco se potencia una mayor cultura investigadora en torno a estas titulaciones.

Esto y la inexistencia de grupos de investigación especializados constituyen un freno a la posibilidad de que haya más tesis doctorales sobre esta materia. De hecho, y según los datos censados por el blog Periodismo Deportivo de Calidad (Rojas-Torrijos, 2013), entre 2001 y 2019 se han defendido 65 tesis doctorales sobre la especialidad en un total de 26 universidades españolas, la mitad de las cuales ni siquiera brindan la asignatura en sus estudios de Grado.

Teniendo en cuenta las limitaciones de la muestra de análisis empleada, al abarcar solo la producción de TFM durante dos años académicos, se trazan futuras líneas de investigación para ampliar el objeto de este estudio con fines exploratorios. Cabe discutir, finalmente, si la producción de TFM puede contribuir a forjar una base válida para impulsar la investigación en este campo en un futuro y si es posible reorientar los planes docentes de estas titulaciones para que, aun no siendo títulos oficiales, puedan propiciar que una mayor transferencia de investigación a la realidad informativa contribuya a una mayor diversidad de temas, enfoques y protagonistas, y sienten las bases para propiciar más sinergias con clubes y medios de comunicación para el desarrollo de estudios y proyectos conjuntos.

Se trataría, en suma, de evaluar cómo la investigación desde la Universidad, incluida la que se fragua dentro de los másteres, puede ayudar a tender puentes entre Academia y profesión periodística para que esa transferencia se materialice en soluciones llegadas desde la especialización e iniciativas periodísticas que sean innovadoras y prósperas.

\section{Bibliografía}

AIMC._Estudio General de Medios (Año Móvil 2019). http://reporting.aimc.es/index.html\#/main/ $\underline{\text { cockpit }}$

ATIC. Miembros de ATIC (Asociación Española de Universidades con Titulaciones de Información y Comunicación). http://titulaciones-atic.com/miembros/

Barlovento Televisión (2019). Análisis televisivo. https://www.barloventocomunicacion.es/wpcontent/uploads/2019/12/analisis-televisivo-2019-BarloventoComunicacion-1.pdf

Cabezuelo, F. y Sotelo, J. (2014). El nuevo panorama del Periodismo Especializado en los estudios de Grado adaptados al EEES. En Esteve, F. y Nieto, J.C. (Eds.). Nuevos retos del periodismo especializado (pp. 35-50). Madrid: Schedas

Cabrera, M., Codina, L. y Salaverria, R. (2019). Qué son y qué no son los nuevos medios. 70 visiones de expertos hispanos. Revista Latina de Comunicación Social, 74, pp. 1506-1520. https:// doi.org/10.4185/RLCS-2018-1396

Castillo, A., Álvarez, A. y Muñiz, J.A. (2013). EEES y la formación en comunicación en España. La estructura de la oferta de posgrado. Estudios del Mensaje Periodístico, 19, pp. 99-110. https:// doi.org/10.5209/rev_ESMP.2013.v19.42015

Díaz-Vázquez, R., García-Díaz, A., Maside, J.M. y Vázquez-Rozas, E. (2018). El Trabajo de Fin de Grado: fines, modalidades y estilos de tutorización. Revista Universitaria de Docencia, 16(2), pp. 159-175. https://doi.org/10.4995/redu.2018.10178 
Elías, C. y Luengo, M. (2014). Periodismo Especializado y áreas de especialización en el currículo de los Grados de Periodismo en las Universidades españolas. En Esteve, F. y Nieto, J.C. (Eds.). Nuevos retos del periodismo especializado (pp. 23-.34). Madrid: Schedas

Haug, G. E. (2015). Grados y másteres en España y el Espacio Europeo de Educación Superior (EEES). CRUE Universidades Españolas: Madrid

Manfredi, J.M., Rojas-Torrijos, J.L. y Herranz, J.M. (2015). Innovación en el periodismo emprendedor deportivo. Modelo de negocio y narrativas. El Profesional de la Información, 24(3), pp. 265-273. https://doi.org/10.3145/epi.2015.may.06

Paniagua, F. y Rojas-Torrijos, J.L. (2015). La oferta actual de másteres de periodismo y comunicación deportiva en las universidades españolas. Una propuesta de mejora. XII Foro Internacional sobre la Evaluación de la Calidad de la Investigación y de la Educación Superior (FECIES). Sevilla, 9-11 de julio.

Pestano, J.M., Rodríguez, C y Del Ponti, P. (2011). Transformaciones en los modelos de formación de periodistas en España. El reto europeo. Estudios sobre el mensaje periodístico, 17(2), pp. 401-415. https://doi.org/10.5209/rev_ESMP.2011.v17.n2.38122

Real Decreto 1393/2007, de 29 de octubre, por el que se establece la ordenación de las enseñanzas universitarias oficiales. https://www.boe.es/eli/es/rd/2007/10/29/1393

Registro de Universidades, Centros y Títulos (RUCT) del Ministerio de Ciencia, Innovación y Universidades. https://www.educacion.gob.es/ruct/home

Rojas-Torrijos, J.L. (10/05/2019). Trabajos Fin de Grado sobre periodismo deportivo en universidades españolas. Periodismo Deportivo de Calidad. https://periodismodeportivodecalidad. blogspot.com/2019/05/trabajos-de-fin-de-grado-sobre_10.html

Rojas-Torrijos, J.L. (02/05/2017). Censo de másteres en Periodismo Deportivo en universidades españolas. Periodismo Deportivo de Calidad. https://periodismodeportivodecalidad.blogspot. com/2017/05/oferta-de-masteres-de-periodismo.html

Rojas-Torrijos, J.L. (10/10/2013). Tesis doctorales sobre periodismo deportivo defendidas en universidades españolas. Periodismo Deportivo de Calidad. https://periodismodeportivodecalidad. blogspot.com/2013/10/tesis-doctorales-sobre-periodismo.html

Sánchez-García, P. (2016). Los efectos de la primera fase del EEES en la enseñanza del periodismo en España: mayor especialización y formación práctica. Communication \& Society, 29(1), pp. 125-143. https://doi.org/10.15581/003.29.1.125-142

Toural, C. y López, X. (2019). Ecosistema de cibermedios en España. Tipologías, iniciativas, tendencias narrativas y desafios. Salamanca: Comunicación Social

Torregrosa, J.F. (2015). Qué formación para qué periodismo. Hacia un currículum a la altura de los retos actuales de la profesión. Índex Comunicación, 5(2), pp. 63-81. 


\section{Anexo}

Relación de los TFM de investigación en másteres de Periodismo Deportivo 2017-2019

\section{Universidad CEU San Pablo-Marca (15)}

- El deporte femenino a través de los medios de comunicación: La imagen de la mujer en Radio Marca

- Rafael Nadal, modelo de héroe deportivo

- El tratamiento del caso de dopaje de Lance Armstrong en la web de Marca

- Origen y desarrollo de la Bundesliga

- El tratamiento del fútbol italiano en Marca y As a raíz de la llegada de Cristiano Ronaldo a Italia

- Santiago Bernabéu y la utilización del Real Madrid por el franquismo

- Origen y desarrollo del Calcio en Italia

- La corrupción en el deporte: el amaño de partidos en el fútbol español

- Elaboración de informe sobre la evolución del futbolista español en el extranjero (a partir de 2000). Causas y posible futuro

- La Champions League: ¿El origen de una identidad supranacional europea?

- La inserción laboral de los deportistas de élite tras el final de su carrera

- Silvio Berlusconi: El deporte al servicio de los intereses mediáticos de la política

- El fenómeno ultra en el fútbol español contemporáneo: despolitización y las gradas jóvenes de animación

- La cobertura televisiva del fútbol en Venezuela: Análisis de los Mundiales de fútbol de 1994 y 2014

- Deporte y ritualidad: El juego de pelota en Mesoamérica

\section{Universidad de Sevilla (13)}

- La mujer en los consejos de administración de los equipos de Primera y Segunda División

- Periodismo deportivo en Youtube

- El papel de la mujer en el arbitraje deportivo. Estudio de caso

- El nuevo formato de los deportes de Antena 3. Estudio del trabajo de la delegación de la cadena en Andalucía.

- Tratamiento radiofónico del fútbol femenino en Sevilla F.C. Radio

- La mediatización del rugby femenino en el Diario de Sevilla y Estadio Deportivo

- Historia y evolución del Supedépor

- El trabajo del fotoperiodista deportivo en el mundo digital contemporáneo

- Comparación entre la Liga Santander de España y la Barclays Premier League de Inglaterra

- La evolución de la locución deportiva en el ámbito del fútbol 
- Análisis del tratamiento mediático de las denuncias sobre discriminación y acoso sexual en la selección femenina de fútbol de Colombia

- El impacto de los derechos televisivos en los clubes profesionales del fútbol andaluz

- El periodista deportivo en Instagram. Análisis de cuatro perfiles profesionales

\section{UEM (6)}

- Análisis del deporte femenino dentro de la sección polideportiva en Marca, As, Mundo Deportivo y sport (febrero 2019-abril 2019)

- Dopaje en el ciclismo, su relación con el periodismo y cómo este afecta a la imagen de un ciclista

- Big Data. Periodismo deportivo de datos

- Fútbol femenino en España: Propuesta para su expansión y promoción desde la comunicación

- La evolución del trato entre el periodista deportivo y el futbolista en España

- Análisis periodístico sobre el marketing y la comunicación del City Football Group. ¿Es realmente una franquicia deportiva?

\section{UCAM (13)}

- La importancia de YouTube y Twitch.tv para los consumidores de eSports. Análisis de consumo por parte de jóvenes jugadores de videojuegos

- Análisis y evolución de las audiencias televisivas de la Fórmula 1 en España (2004-2018)

- La dimensión política del deporte en la prensa y el ocio como mecanismo de control en el Alicante de Primo de Rivera: El ejemplo de la I Olimpiada Levantina de 1924

- La imagen del árbitro en los medios de comunicación

- La responsabilidad social de la prensa digital deportiva española: análisis de calidad y malas prácticas

- La influencia de las redes sociales durante la emisión en radio de un evento deportivo: análisis del uso de Twitter durante la retrasmisión de un partido de fútbol

- La responsabilidad del periodismo deportivo con la violencia en el deporte: una revisión teórica sobre el fenómeno

- El deporte femenino en España: un estado de la cuestión desde el punto de vista de cinco deportistas gallegas de élite

- Análisis del tratamiento mediático del dopaje en la prensa deportiva española. La cobertura del caso Lance Armstrong en los periódicos MARCA y El Mundo

- La evolución del fútbol femenino: perspectivas sobre su auge en los medios y entre los aficionados y aficionadas

- Análisis del tratamiento informativo, mediático y deportivo de la gimnasia rítmica en España

- La presencia de la mujer en el deporte y en el periodismo deportivo

- El tratamiento de la figura de Pep Guardiola en las ediciones digitales de los principales periódicos deportivos españoles 